\title{
Correction to: Analysis of T cell receptor clonotypes in tumor microenvironment identifies shared cancer-type-specific signatures
}

\author{
Yvonne H. F. Teng ${ }^{1,2,3}$ (1) Hong Sheng Quah" ${ }^{1,3}$. Lisda Suteja ${ }^{1,2}$ • João M. L. Dias ${ }^{4}$ Annalisa Mupo ${ }^{5}$ \\ Rachael J. M. Bashford-Rogers ${ }^{6}$. George S. Vassiliou ${ }^{7} \cdot$ Melvin L. K. Chua ${ }^{3,8}$ • Daniel S. W. Tan ${ }^{1,2,3}$. \\ Darren W. T. Lim ${ }^{2,3,9} \cdot$ N. Gopalakrishna lyer ${ }^{1,3,10}$
}

Published online: 22 October 2021

(c) Springer-Verlag GmbH Germany, part of Springer Nature 2021

\section{Correction to: Cancer Immunology, Immunotherapy https://doi.org/10.1007/s00262-021-03047-7}

The original version of this article unfortunately contained a mistake. Accession study number was incorrect in the section "Availability of data and materials".

The "Availability of data and materials" section should read as:

Availability of data and materials Sequence data have been deposited at the European Genome-Phenome Archive (EGA), which is hosted by the EBI and the CRG,

The original article can be found online at https://doi.org/10.1007/ s00262-021-03047-7.

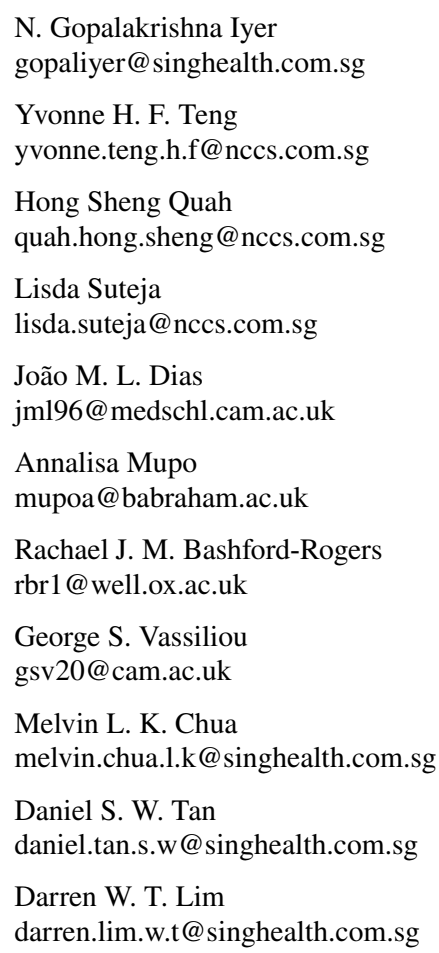

under accession study number EGAS00001005480 (to be released upon acceptance). Further information about EGA can be found on https://ega-archive.org and "The European Genome-Phenome Archive of human data consented for biomedical research" (http://www.nature.com/ng/journal/ v47n7/full/ng.3312.html). Custom scripts to run the analyses described in the manuscript are available at https://github. com/yhfteng/Shared-TCRs-in-cancers.

Publisher's Note Springer Nature remains neutral with regard to jurisdictional claims in published maps and institutional affiliations.

1 Cancer Therapeutics Research Laboratory, National Cancer Centre Singapore, 11 Hospital Crescent, Singapore 169610, Singapore

2 Division of Medical Oncology, National Cancer Centre Singapore, Singapore, Singapore

3 Duke-NUS Medical School, Singapore, Singapore

4 Hutchison/MRC Research Centre, MRC Cancer Unit, University of Cambridge, Cambridge Biomedical Campus, Cambridge CB2 0XZ, UK

5 Babraham Research Institute, Cambridge CB22 3AT, UK

6 Wellcome Trust Centre for Human Genetics, University of Oxford, Roosevelt Dr, Headington, Oxford OX3 7BN, UK

7 Wellcome-MRC Cambridge Stem Cell Institute, Jeffrey Cheah Biomedical Centre, University of Cambridge, Cambridge Biomedical Campus, Puddicombe Way, Cambridge CB2 0AW, UK

8 Division of Radiation Oncology, National Cancer Centre Singapore, Singapore, Singapore

9 Institute of Molecular and Cell Biology, A-STAR, Singapore, Singapore

10 Department of Head and Neck Surgery, National Cancer Centre Singapore, Singapore, Singapore 